2. There is significant heterogeneity in the current guidelines despite BSG committee advice on guideline writing.

These findings risk reducing the utility of these guidelines and highlight important areas of unmet research need that should be addressed as a matter of priority. Studies co-ordinated through locoregional networks could substantially improve the evidence base and ultimately patient outcomes.

Competing interests None declared.

\section{PMO-020 AUDIT TO ASSESS THE ACCEPTABILITY AND USEFULNESS OF AN AUDIOVISUAL AID IN THE PREPARATION OF PATIENTS FOR OUTPATIENT ENDOSCOPY}

doi:10.1136/gutjnl-2012-302514b.20

${ }^{1}$ I Williams, ${ }^{*}$ S Edwards, ${ }^{1} \mathrm{R}$ Boulton. ${ }^{1}$ Department of Gastroenterology, OEHB, Birmingham, UK; ${ }^{2}$ Clinical Photography, OEHB, Birmingham, UK

Introduction Written information is traditionally used to educate patients about endoscopy, but it is difficult to fully convey endoscopy via a leaflet. We assessed whether a video following a "patient" through the endoscopy journey was a useful tool to assist patient's understanding of endoscopy as a supplement to the information that we have been using in our Unit.

Methods A DVD showing the endoscopy process was sent to all individuals booked for an outpatient upper GI endoscopy over 2 months. On arrival at endoscopy unit they were asked to complete a questionnaire.

Results Abstract PMO-020 table 1 demonstrating responses to questions 4-6. Of 158 questionnaires, 149 replies were suitable for analysis. $81 \%$ gave the DVD top score for clarity and ease of understanding. The majority of responders found the DVD useful in preparing them for what will happen on the day of endoscopy with $81 \%$ of responders scoring the DVD one of the top two ratings for this category. All of the responders felt the DVD was a good idea and all but one individual recommended that we should continue to include it in the information packs.

\section{Abstract PM0-020 Table 1}

\begin{tabular}{llll}
\hline $\begin{array}{l}\text { Score out } \\
\text { of } 5\end{array}$ & $\begin{array}{l}\text { Do you find the } \\
\text { DVD clear and easy } \\
\text { to understand? }\end{array}$ & $\begin{array}{l}\text { Did You find the } \\
\text { DVD interesting } \\
\text { to watch? }\end{array}$ & $\begin{array}{l}\text { Did you find it useful } \\
\text { in preparing you for } \\
\text { what will happen on } \\
\text { the day? }\end{array}$ \\
\hline 5 & 67 & 52 & 58 \\
4 & 9 & 15 & 9 \\
3 & 3 & 9 & 9 \\
2 & 1 & 3 & 2 \\
1 & 3 & 4 & 3 \\
0 & 0 & 0 & 2 \\
\hline
\end{tabular}

Conclusion Overall, the response to the endoscopy DVD was very positive. Some of the issues related to not watching the DVD will likely be solved once it is in use in an endoscopy pre-admission clinic. On the basis of this extremely positive result, we plan to utilise the endoscopy information DVD more widely and extend it to other endoscopic procedures.

Competing interests None declared.

\section{PM0-021 OPTIMISING OUT-PATIENT PARENTERAL IRON ADMINISTRATION USING TOTAL DOSE INFUSIONS}

doi:10.1136/gutjnl-2012-302514b.21

${ }^{1} \mathrm{~J}$ Swabe, ${ }^{2} \mathrm{C}$ Wijayasekara, ${ }^{1} \mathrm{~J}$ Allen, ${ }^{2} \mathrm{~F}$ Cummings. ${ }^{1}$ Department of Pharmacy, University Hospital Southampton NHS Foundation Trust, Southampton, UK;
${ }^{2}$ Department of Gastroenterology, University Hospital Southampton NHS Foundation Trust, Southampton, UK

Introduction Iron deficiency is common in the inflammatory bowel disease population $\left(36 \%-90 \%{ }^{1}\right)$ and is often poorly managed. Guidelines on the management of iron deficiency anaemia recommend that parenteral iron should be used if oral iron supplementation is either ineffective or not tolerated. ${ }^{1}$ Iron Sucrose (IS, Venofer ${ }^{\mathbb{B}}$ ) infusions take on average $85 \mathrm{~min}$ to be administered and require between 5 and 13 infusions for a therapeutic dose of iron to be given Ferric Carboxymaltose (FC, Ferinject ${ }^{\circledR}$ ) is a total dose infusion parenteral iron preparation. It requires $1-2$ infusions for therapeutic dose delivery and allows shorter administration times. We audited the outcomes of switching from IS to FC in terms of therapeutic efficacy and patient experience.

Methods Current practice with IS was prospectively audited over a 6-week period. All admissions for parenteral iron to the Managed Care Unit (MCU) were included. Nursing staff completed a questionnaire including patient demographics, indication and record the patient journey. Patients completed a satisfaction survey. FC was introduced in early December 2011 and data collected for a further 6-week period. FC was dosed according to the summary of product characteristics, updated 25 October 2011.

Results Data are presented as median (range) unless otherwise stated. All patients in the FC group and $45 \%$ in the IS group were cannulated at the first attempt. No patients in the FC group and $20 \%$ in the IS group reported severe pain on cannulation. All patients in the FC group and $83 \%$ in the IS group received their full course. It took a median -days (range) of $49(30-113)$ for the IS patients and 9 (8-10) for the FC patients to complete their treatment course. Two patients in the FC group who had previously had IS commented on the advantage of fewer infusions. In both groups over $70 \%$ of patients rated their experience as good or very good.

Conclusion This real life data shows clear advantages of using a TDI parenteral iron preparation. A reduction in the number of admissions and time spent in the MCU is demonstrated with more patients receiving the recommended dose in a shorter time. Efficiency can be improved by advanced prescribing and our data may show gaps in the knowledge of prescribers. Patient satisfaction remained high with FC.

\section{Abstract PM0-021 Table}

\begin{tabular}{lll}
\hline & Iron sucrose & Ferric carboxymaltose \\
\hline Number of admissions/patients & $51 / 26$ & $16 / 15$ \\
Mean age & 61 & 50 \\
Patient group (gastro/renal/other\%) & $62 / 31 / 7$ & $53 / 47 / 0$ \\
Prescribed in advance \% & 88 & 31 \\
Time to prescribing-min & $90(40-120)$ & $20(15-300)$ \\
Mean infusion time-min & 85 & 23 \\
Number of infusions & $6(5-13)$ & $1(1-2)$ \\
Length of treatment course-days & $49(30-113)$ & $9(1-10)$ \\
Received full treatment course \% & 86 & 100 \\
\hline
\end{tabular}

Competing interests J Swabe: None Declared, C Wijayasekara: None declared, J Allen: None declared, F Cummings speaker bureau with: Vifor Pharmaceuticals.

\section{REFERENCE}

1. Gomollón F, Gisbert JP. Anemia and inflammatory bowel diseases. World $J$ Gastroenterol. 2009:15:4659-65. 